possible complications. However, incomplete resection of the hematoma resulted in uncontrollable bleeding from the subcapsular lesion or recurrence of hematoma within several years in some publications. ${ }^{2,7}$ Complete extirpation, including the capsule, would be desired for cure.

In our patient acute massive hemoptysis was treated with emergency tracheal intubation, balloon occlusion of the bleeding bronchus, and bronchial artery embolization. In the literature neither massive hemoptysis nor emergency management for chronic expanding hematoma of the thorax has been reported. Because the bleeding spread to the contralateral lung, the operation was intentionally delayed until the remaining lung function recovered. Thereafter, the patient successfully underwent a pneumonectomy with extirpation of the hematoma. Division of the hilum first and sequential distal dissection of the lung and hematoma through a median sternotomy might have worked well for bleeding control.

\section{References}

1. Iuchi K, Inada K, Yamamoto M, Ichimiya A, Tada H, Mori T, et al. Analysis of the empyema without pus (organized empyema). Jpn Chest Surg. 1998;2-11-7.

2. Hanagiri T, Muranaka H, Hoshimoto M, Nishio T, Sakai S, Ono M, et al. Chronic expanding hematoma in the chest. Ann Thorac Surg. 1997; 64:559-61.

3. Roper CL, Cooper JD. Chronic expanding hematoma of the thorax. J Thorac Cardiovasc Surg. 2001;122:1046-8.

4. Hwang GL, Moffatt SD, Mitchell JD, Leung AN. Chronic expanding hematoma of the thorax. AJR Am J Roentgenol. 2003;180:1182-3.

5. Reid JD, Kommareddi S, Lankerani M, Park MC. Chronic expanding hematomas. A clinicopathologic entity. JAMA. 1980;244:2441-2.

6. Labadie EL, Glover D. Physiopathogenesis of subdural hemangiomas. Part 1: Histological and biochemical comparisons of subcutaneous hematoma in rats with subdural hematoma in man. J Neurosurg. 1976;45:382-92.

7. Matsuge S, Hosokawa Y, Yamzaki S, Sato K, Murakami Y, Yamakawa $\mathrm{T}$, et al. Five cases of surgically resected chronic expanding hematoma in the chest. Jpn J Thorac Surg. 2000;53:768-73.

\title{
Thyroid metastasis after resection of atypical bronchial carcinoid
}

\author{
Pier Luigi Filosso, MD, ${ }^{\text {a } A n n a ~ A r s l a n i a n, ~ M D, ~}{ }^{a}$ Nicola Palestini, MD, ${ }^{\mathrm{b}}$ Alessandra Farnetti, MD, ${ }^{\mathrm{b}}$ Mauro Papotti, MD, ${ }^{\mathrm{c}}$ \\ Massimo Bongiovanni, MD, ${ }^{\mathrm{c}}$ Roberto Giobbe, MD, and Enrico Ruffini, MD, ${ }^{a}$ Torino, Italy
}

B ronchial carcinoids $(\mathrm{BCs})$ are rare and constitute less than $2 \%$ of pulmonary tumors. ${ }^{1}$ They are characterized by slow, mainly endobronchial growth, with infrequent regional lymph node involvement or distant metastases. ${ }^{2}$ Atypical carcinoids are part of the spectrum of neuroendocrine bronchopulmonary tumors, according to the 1999 World Health Organization-International Association for the Study of Lung Cancer (WHO-IASLC) Lung Tumor classification. ${ }^{3}$ They present 2 low-grade (typical and atypical carcinoids) and 2 high-grade malignant varieties (large cell neuroendocrine carcinoma and small cell lung carcinoma), the latter characterized by a high tendency toward mediastinal and distant metastatic spread.

Among low-grade malignant tumors, atypical carcinoids show a more aggressive biologic behavior than typical carcinoids ${ }^{4}$ : mediastinal lymph node metastases occur at presentation in about $15 \%$ of cases. Distant metastases are generally in the liver and in the bone.

From the Department of Thoracic Surgery, Division of General Surgery, and the Department of Human Oncology and Biomedical Sciences, University of Torino, Torino, Italy.

Received for publication Feb 11, 2003; accepted for publication March 12, 2003.

Address for reprints: Pier Luigi Filosso, MD, University of Torino, San Giovanni Battista Hospital, Via Genova, 3, Torino 10126, Italy (E-mail: pierluigifilosso@tiscalinet.it).

J Thorac Cardiovasc Surg 2004;127:1840-3

$0022-5223 / \$ 30.00$

Copyright $\odot 2004$ by The American Association for Thoracic Surgery

doi:10.1016/j.jtcvs.2003.03.005
We present a case of a woman in whom thyroid metastasis occurred 30 months after the resection of an atypical BC.

\section{Clinical Summary}

A 53-year-old white woman was referred to us in June 1999 because of the presence of a large pulmonary mass $(7 \times 3 \mathrm{~cm}$ in size) in the right lower lobe. The lesion had been detected with chest radiography performed in July 1998, and it was $5 \times 2 \mathrm{~cm}$ in size, but at that time, the patient refused the intervention. She eventually agreed to be operated on because of the progression of the lesion and the associated cough and dyspnea. A preoperative transthoracic fine-needle aspiration biopsy was performed, and neoplastic cells with neuroendocrine features were observed. ${ }^{111}$ In-DTPA-Pentetreotide scintigraphy (Octreoscan) was performed for a correct preoperative assessment, resulting in an elective uptake in correspondence of the pulmonary lesion with no other abnormal uptakes. Chromogranin A and neuron-specific enolase serum levels were performed and increased: $197 \mathrm{ng} / \mathrm{mL}$ (normal values, $20-100 \mathrm{ng} / \mathrm{mL}$ ) and $62 \mathrm{ng} / \mathrm{mL}$ (normal values, $<12.5 \mathrm{ng} / \mathrm{mL}$ ), respectively.

The patient underwent a right pneumonectomy with systemic lymphadenectomy: a more conservative intervention was not possible because of the tumor size. A residual tumor was left on the inferior pulmonary vein stump.

Grossly, an 8-cm mass with poorly demarcated borders infiltrating the mediastinal pleura and the adipose mediastinal tissue was found. The cut surface was tan-yellow, and a brown lymph node was adherent and directly infiltrated by the tumor.

Microscopically, the tumor was not capsulated, and it had a uniform organoid growth pattern composed of nests and sheets of cuboidal and eosinophilic cells. Rosette-like structures were also 

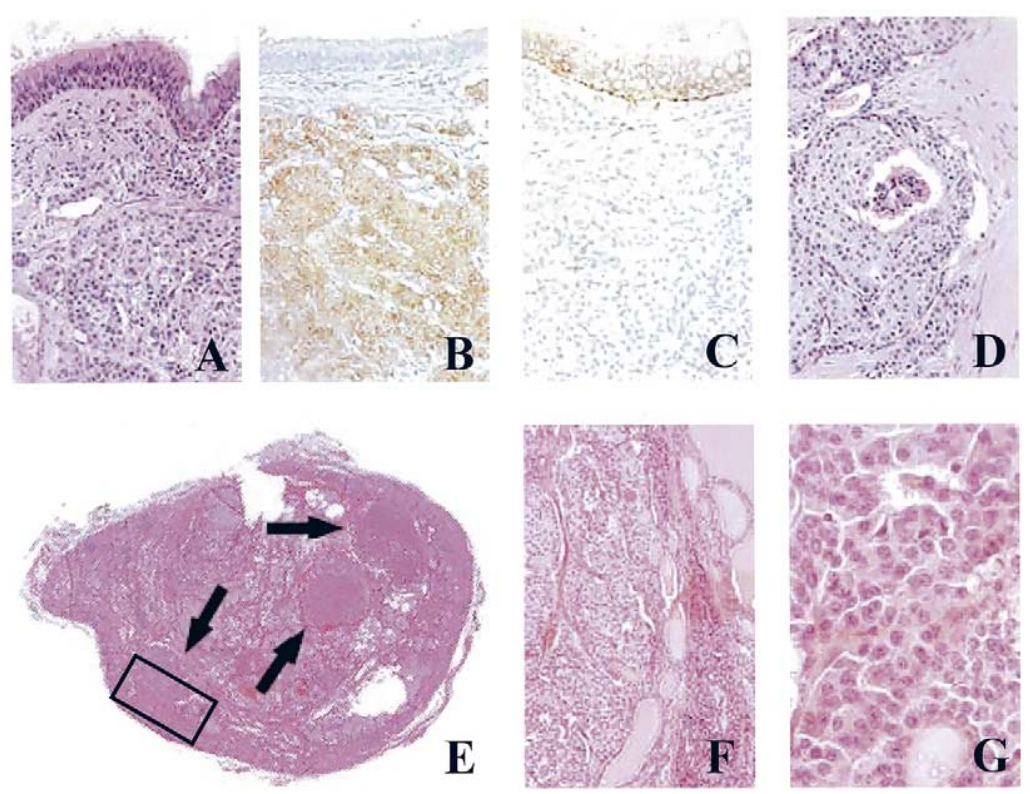

Figure 1. A-D, Pulmonary tumor: nests and sheets of polygonal eosinophilic cells are arranged in the classical organoid pattern of pulmonary carcinoids, infiltrating the bronchial epithelium (top, $A)$. The neuroendocrine nature of this proliferation is confirmed by means of immunohistochemical positivity for Chromogranin $A(B)$ and by the absence of 34BE12 cytokeratin immunoreactivity in neoplastic cells (as opposed to the staining of nonneoplastic bronchial epithelium; C). A small focus of necrosis is also present (D). E-G, Thyroid metastases: a cross-section of the right thyroid lobe showed small multiple nodules in the parenchyma (arrows, E). Normal follicles are infiltrated by neuroendocrine tumor cells arranged in small cords and nests, as seen in the primary pulmonary tumor ( $F$ and G).

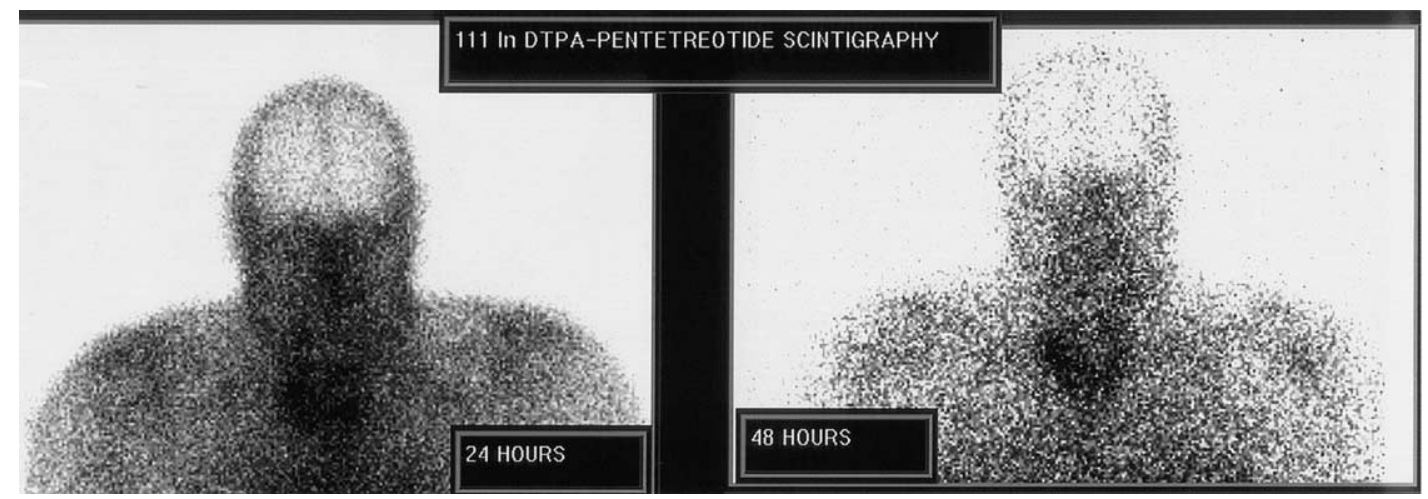

Figure 2. Postoperative ${ }^{111}$ In-DTPA-Pentetreotide scintigraphy (Octreoscan) showing an elective uptake at the right thyroid lobe.

evident. The nuclei had either finely granular or coarse chromatin, and nucleoli were occasionally present (Figure 1, A). The inferior pulmonary vein was infiltrated by neoplastic cells. Limited foci of necrosis were evident, and the mitotic count was 3 per 10 highpower fields. One peribronchial lymph node and the visceral pleura were directly infiltrated by the tumor.

Immunohistochemically, the cells were strongly positive for chromogranin A (clone LK2H10; Dako, Glostrup, Denmark) and negative for high-molecular-weight cytokeratin (clone 34BE12,
Dako; Figure 1, $B-D$ ). The proliferation index (as expressed by positivity for Mib-1 monoclonal antibody [Immunotech, Marseille, France]) was $6 \%$ in neoplastic cells.

Pathologic diagnosis was pT4 N1 atypical carcinoid or welldifferentiated neuroendocrine carcinoma. The postoperative course was uneventful.

Medical therapy with Octreotide (Sandostatina LAR $30 \mathrm{mg}$; Novartis Pharma SPA, Origgio, Italy) was started and followed by mediastinal adjuvant radiotherapy. 
A strict follow-up was planned with thoracic and upper abdominal computed tomographic scanning, Octreoscan, serum chromogranin A, and neuron-specific enolase dosages.

In December 2001, Octreoscan showed an uptake in the right thyroid lobe (Figure 2); an ultrasonographic evaluation demonstrated the presence of a $2 \times 1-\mathrm{cm}$ nodule. A fine-needle aspiration biopsy was performed: the highly cellular smears contained medium-sized polygonal and eosinophilic neoplastic cells with finely granular chromatin and faint nucleoli. These cells were unreactive for thyroglobulin and calcitonin and positive for chromogranin A. Diagnosis of thyroid metastasis of neurendocrine carcinoma of the lung was thus made.

The patient underwent a radical thyroidectomy and cervical lymphadenectomy. Grossly, the thyroid weighed $41 \mathrm{~g}$, with multiple bilateral red-whitish nodules 0.5 to $1.5 \mathrm{~cm}$ in size.

Microscopically, these nodules were composed of polygonal and eosinophilic cells arranged in cords and nests, having the typical organoid pattern of carcinoid tumors (Figure 1, E-G). Foci of necrosis were observed. No thyroglobulin immunostaining (polyclonal; BioGenex, San Ramon, Calif) was seen. The absence of calcitonin immunoreactivity (polyclonal, Dako) also ruled out the diagnosis of medullary thyroid carcinoma. Chromogranin A immunostaining (clone LK2H10, Dako) was strongly positive. The morphologic features and the similarity with the previously resected pulmonary neuroendocrine lesion confirmed the preoperative diagnosis of metastatic neuroendocrine carcinoma.

The postoperative course was also uneventful.

The patient is alive and well without recurrence 13 months after the second intervention, and no adjuvant chemotherapy or radiotherapy was planned; she is now only continuing medical therapy with Octreotide (Sandostatina LAR $30 \mathrm{mg}$; Novartis Pharma, Origgio, Italy), which is well tolerated without important side effects.

\section{Discussion}

BCs have raised important issues for thoracic surgeons, in particular whether to regard them as benign or malignant tumors. BCs are a part of the spectrum of the neuroendocrine tumors of the lung ${ }^{3}$ : the WHO-IASLC classification considers BCs as different from large cell neuroendocrine carcinoma and small cell lung carcinoma on the basis of their histologic and biologic features. $\mathrm{BCs}$ are low-grade malignant tumors that can be locally invasive or spread to mediastinal lymph nodes, but distant metastases are rare. $^{4}$ Martini and colleagues ${ }^{2}$ report that only $10 \%$ to $15 \%$ of patients present with regional lymph node metastases at diagnosis. Surgical intervention represents the treatment of choice, even if mediastinal nodal metastases are present: BCs are generally unresponsive to preoperative radiation or chemotherapy, and long-term prognosis is good for both the typical and atypical forms when treated with radical resection. ${ }^{5}$

Distant metastases are rare: we experienced liver metastases in 7 patients with radically resected atypical $\mathrm{BCs},{ }^{5,6}$ in whom carcinoid syndrome was the first symptom. Octreoscan was effective in diagnosis, and octreotide therapy allowed us to control symptoms and to reduce metastases in size in 2 cases. $^{6}$

We point out the importance of ${ }^{111}$ In-DTPA-Pentetreotide scintigraphy (Octreoscan) in the early diagnosis of the thyroid metastasis and in general in the follow-up of neurorendocrine lung tumors. $^{7,8}$ Neuroendocrine tumors in vitro express somatostatin receptor subtype 2, which can be imaged in vivo by using Octreoscan.

Octreoscan has demonstrated its effectiveness in diagnosis of the primary tumor but particularly in detecting early recurrences or distant metastases in asymptomatic patients earlier than with traditional radiologic procedures. ${ }^{9}$

A positive Octreoscan result should guide an effective medical therapy with somatostatin analogs (octreotide and lanreotide). ${ }^{6}$

Positron emission tomography scanning in the presence of BCs shows lower uptake than in non-small cell lung cancers; therefore positron emission tomography scanning is, at present, not indicated for the differential diagnosis of a solitary pulmonary nodule with neuroendocrine features from a benign process. ${ }^{10}$

Complete resection with radical mediastinal lymph node dissection provides excellent local control and long-term survival in $\mathrm{BCs}$; the presence of mediastinal metastases does not preclude surgical intervention. ${ }^{2}$

Local recurrences are rare and generally associated with an inadequate (not anatomic) surgical treatment of the primary tumor. Distant metastases appear to be related to the histologic form of BCs (atypical carcinoid) more than to the nodal status. ${ }^{2,11}$ When possible, surgical intervention remains the treatment of choice for recurrences because there is no evidence that radiotherapy or chemotherapy are effective in local control or in long-term survival.

Our experience provides evidence that the use of somatostatin analogs (octreotide and lanreotide) is effective in cases of distant metastases discovered by means of Octreoscan ${ }^{6}$ in which surgical intervention is not feasible. Octreotide binds with high affinity to somatostatin receptor subtype 2 , which is expressed in neuroendocrine tumoral cells. Octreotide has demonstrated a valid inhibitory growth effect on neoplastic cells, and it is well tolerated, without important side effects. The optimal dose of octreotide is generally $1500 \mathrm{mg} / \mathrm{d}$ subcutaneously, but a recent long-acting form (Octreotide LAR) appears more simple for patient management because only one dose every 28 days is necessary.

\section{References}

1. Harpole D, Feldman J, Buchanan S, Young W, Wolfe W. Bronchial carcinoid tumors: a retrospective analysis of 126 patients. Ann Thorac Surg. 1992;45:50-5.

2. Martini N, Zaman M, Banis M, Burt M, McCormack P, Rush V, et al. Treatment and prognosis in bronchial carcinoid involving regional lymph nodes. J Thorac Cardiovasc Surg. 1994;107:1-7.

3. Travis WD, Corrin B, Shimosato Y, Brambilla E. The histological typing of lung and pleural tumors. 3rd ed. Berlin: Springer Verlag; 1999.

4. Marty-Ané CH, Costes V, Pujol JL, Alauzen M, Baldet P, Mary H. Carcinoid tumors of the lung: do atypical features require aggressive management? Ann Thorac Surg. 1995;59:78-83.

5. Filosso PL, Rena O, Donati G, Casadio C, Ruffini E, Papalia E, et al. Bronchial carcinoid tumors: surgical management and long-term outcome. J Thorac Cardiovasc Surg. 2002;123:303-9.

6. Filosso PL, Ruffini E, Oliaro A, Papalia E, Donati G, Rena O. Long-term survival in metastatic atypical bronchial carcinoid treated with octreotide. Eur J Cardiothorac Surg. 2002;21:913-7.

7. Ferguson MK, Landreneau RJ, Hazelrigg SR, Altorky NK, Naunheim KS, Zwischenberger JB, et al. Long-term outcome after resection for bronchial carcinoid tumors. Eur J Cardiothorac Surg. 2000;18:156-61.

8. Oliaro A, Filosso PL, Bellò M, Casadio C, Angusti T, Masaneo I, et al. 
Use of ${ }^{111}$ In-DTPA-octreotide scintigraphy in the diagnosis of neuroendocrine and non-neuroendocrine tumors of the lung. Preliminary results. J Cardiovasc Surg. 1997;38:313-5.

9. Musi M, Carbone RG, Bertocchi C, Cantalupi DP, Pugliese C, Virotta G. Bronchial carcinoid tumors: a study on clinicopathological features and role of octreotide scintigraphy. Lung Cancer. 1998; 22:97-102.
10. Erasmus JJ, McAdams HP, Patz EF Jr, Coleman RE, Ahuja V, Goodman PC. Evaluation of primary pulmonary carcinoid tumors using FDG PET. AJR Am J Roentgenol. 1998;170:1369-73.

11. El Jamal M, Nicholson AG, Goldstraw P. The feasibility of conservative resection for carcinoid tumours: is pneumonectomy even necessary for uncomplicated cases? Eur J Cardiothorac Surg. 2000;18: 301-6.

\title{
An autopsy case of angiosarcoma arising around a woven Dacron prosthesis after a Cabrol operation
}

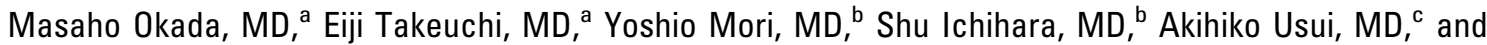 \\ Yuichi Ueda, MD, ${ }^{\mathrm{C}}$ Nagoya, Japan
}

\begin{abstract}
Clinical Summary
A 50-year-old man who was given a diagnosis of Marfan syndrome was hospitalized because of a right visual field defect. $\mathrm{He}$ had undergone a Cabrol operation for annuloaortic ectasia and aortic valve regurgitation 17 years earlier. His ascending aorta and aortic root were replaced with a woven Dacron graft (DeBakey Vasculour; C.R. Bard, Inc, Murray Hill, NJ) and a mechanical valve (Björk-Shiley monostrut heart valve, $25 \mathrm{~mm}$; Shiley, Inc, Irvine, Calif). He underwent mitral valve replacement with a mechanical valve for mitral valve insufficiency caused by acute endocarditis 5 years earlier. He was hospitalized because of rightside hemiparesis but fell into a semicoma thereafter. Computed tomography $(\mathrm{CT})$ revealed a left occipital brain tumorous shadow. A tumorectomy of the left occipital lobe was performed, but the patient died as a result of an expanding brain hemorrhage 2 weeks later. The brain tumor was diagnosed as metastatic angiosarcoma.
\end{abstract}

\section{Autopsy Findings}

The aortic root had a wrap inclusion of the aneurysmal sac surrounding a woven Dacron aortic graft. There was a tumorous mass along the noncoronary cusp of the anastomosed site, with soft granulation tissue in it (Figure 1). The tumor extended into the aneurysmal sac and invaded the graft inside as well. The brain had multiple metastatic tumors and expanding hemorrhaging. There were no metastatic tumors in the other organs.

From the Departments of Cardiovascular Surgery ${ }^{\mathrm{a}}$ and Pathology, ${ }^{\mathrm{b}}$ Nagoya National Hospital, and the Department of Cardiovascular Surgery, ${ }^{\mathrm{c}}$ Nagoya University Graduate School of Medicine, Nagoya, Japan.

Received for publication Nov 1, 2003; revisions requested Dec 24, 2003 accepted for publication Jan 8, 2004.

Address for reprints: Masaho Okada, MD, Department of Cardiovascular Surgery, Nagoya University School of Medicine, 65 tsurumaicho, Shouwaku, Nagoya, Aichi 466-8560, Japan (E-mail: OKMASAHO@aol.com).

J Thorac Cardiovasc Surg 2004;127:1843-5

$0022-5223 / \$ 30.00$

Copyright $\odot 2004$ by The American Association for Thoracic Surgery

doi:10.1016/j.jtcvs.2004.01.021
Microscopic examination revealed a moderately differentiated angiosarcoma arranged in complex anastomosing channels and irregular sheets (Figure 2).

Neoplastic endothelial cells proliferated on the Dacron graft, mainly outside but also inside. Neoplasmic cells were also observed among individual Dacron fibers. There were poorly cohesive neoplasmatic cells in the wrap inclusion of the aneurysmal sac. Among immunohistochemical markers for endothelial differentiation, only CD31 was positive; CD34 and factor VIII were both negative.

\section{Discussion}

Foreign bodies have been shown to induce sarcomas in experimental animals, ${ }^{1}$ but it has only rarely been reported in human subjects that sarcomas have developed adjacent to foreign material introduced into the body either iatrogenically or accidentally. ${ }^{2}$ In thoracic surgery various forms of Dacron prostheses are commonly used. Oppenheimer and colleagues ${ }^{1}$ briefly mentioned that the common denomination of tumor-producing materials was a long-chain polymer structure, such as Dacron. Seven sarcomas have been reported arising in association with Dacron vascular prostheses in the English-language literature. ${ }^{3-6}$ The present case is angiosarcoma arising from the anastomosed site of the aortic root with a Dacron aortic graft. Neoplastic cells strongly expressed CD31, a reliable marker that has both relative specificity and excellent sensitivity to angiosarcomas of all types, ${ }^{7,8}$ confirming the pathologic diagnosis. Neoplastic cells infiltrated both sides of the endothelial layer of the graft from the anastomosed site. Therefore we speculate that the anastomosed site with a Dacron graft is the origin of the angiosarcoma. Angiosarcoma has also been reported to have developed at the site of defunctionalized arteriovenous fistulas. ${ }^{9,10}$ The wrap inclusion of the aneurysmal sac around the aortic root is one example of a defunctionalized arteriovenous fistula. There was a thrombus with neoplastic cells in it. An exuberant host response around the foreign material might represent an important intermediate step in the development of the sarcoma. ${ }^{11}$ The cells in the inflammatory or repair process rarely undergo a malignant transformation, which is probably associated with an oncogene activation and a tumor suppressor gene inacti- 